\title{
Levels and correlates of nutritional status of women of childbearing age in rural Bangladesh
}

\author{
Rasheda Khanam ${ }^{1, *}$, Anne Shee CC Lee ${ }^{2}$, Malathi Ram ${ }^{1}$, MA Quaiyum ${ }^{3}$, Nazma Begum ${ }^{4}$, \\ Allysha Choudhury ${ }^{5}$, Parul Christian ${ }^{6}$, Luke C Mullany ${ }^{1}$ and Abdullah H Baqui ${ }^{1}$ for the MIST \\ Study Team of the Projahnmo Study Group in Bangladesh \\ ${ }^{1}$ International Center for Maternal and Newborn Health, Department of International Health, Johns Hopkins \\ Bloomberg School of Public Health, 615 N. Wolfe Street, Suite E-8624, Baltimore, MD 21205, USA: ${ }^{2}$ Department of \\ Pediatric Newborn Medicine, Brigham and Women's Hospital, Boston, MA, USA: ${ }^{3}$ International Centre for

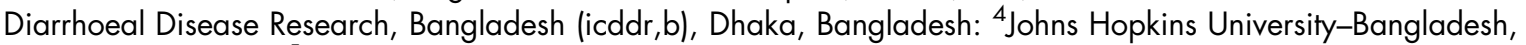 \\ Dhaka, Bangladesh: ${ }^{5}$ Department of Epidemiology, Johns Hopkins Bloomberg School of Public Health, Baltimore, \\ MD, USA: ${ }^{6}$ Bill and Melinda Gates Foundation, Seattle, WA, USA
}

Submitted 11 September 2017: Final revision received 20 April 2018: Accepted 6 July 2018: First published online 15 August 2018

\begin{abstract}
Objective: The present study examined the prevalence of and risk factors for malnutrition in a population-based cohort of women of childbearing age in rural Bangladesh.

Design: A cross-sectional study that collected pre-pregnancy weight, height, and data on selected risk factors for nutritional status of women.

Setting: The study was conducted in Sylhet District of Bangladesh.

Subjects: Study subjects included 13230 non-pregnant women of childbearing age. Women were classified into underweight $\left(<18.5 \mathrm{~kg} / \mathrm{m}^{2}\right)$, normal $\left(18.5-24.9 \mathrm{~kg} / \mathrm{m}^{2}\right)$ and overweight/obese $\left(\geq 25 \cdot 0 \mathrm{~kg} / \mathrm{m}^{2}\right)$ using BMI; and into moderate to severe stunting $(<150 \mathrm{~cm})$, mild stunting $(150-<155 \mathrm{~cm})$ and normal $(\geq 155 \mathrm{~cm})$ using height. Two multinomial logistic regression models were fitted for BMI: model 1 examined individual and household factors associated with BMI, and model 2 additionally examined the association of community variables. The same analysis was conducted for height.

Results: Prevalence of underweight, overweight/obesity and moderate to severe stunting was 37.0, $7 \cdot 2$ and $48 \cdot 6 \%$, respectively. Women's education and household wealth were inversely related to both underweight status and stunting. Underweight rate was significantly lower in the post-harvest season. Women with any education and who belonged to households with higher wealth were more likely to be overweight/obese.

Conclusions: The study documented high underweight and stunting, and moderate overweight/obesity rates among rural Bangladeshi women; and recommends design and implementation of a multidimensional intervention programme based on individual-, household- and community-level risk factors that can address underweight, stunting and overweight/obesity to improve the nutritional status of women of childbearing age in Bangladesh.
\end{abstract}

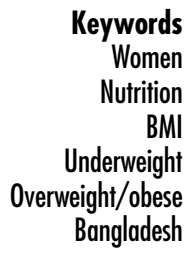

The 2008 Lancet maternal and child nutrition series quantified the global prevalence of maternal undernutrition, predicted its short- and long-term consequences, and estimated the potential for reducing the burden through high and equitable coverage of proven nutrition interventions $^{(1-4)}$. Five years after the initial series, a second series re-evaluated the underlying factors of maternal and child malnutrition and examined the growing concern of overweight and obesity for women and their consequences in low- and middle-income countries. Many of these countries are experiencing the double burden of malnutrition: continued undernutrition along with the emerging problem of overweight and obesity ${ }^{(2,5-7)}$.

The burden of maternal undernutrition continues to be high in South Asia and parts of Africa. In South Asia, the prevalence of maternal undernutrition, both acute and chronic, ranges from 10 to $40 \%^{(8)}$. BMI is an important indicator of the nutritional status of a population. The 
proportion of women reported to be underweight in most low- and middle-income countries ranges from 10 to $19 \%{ }^{(2,5)}$. Stunting is a marker of chronic undernutrition $^{(9,10)}$ and is driven by genetic and environmental factors ${ }^{(11,12)}$. The prevalence of maternal underweight status and stunting is high in Bangladesh; about a third of ever-married women are underweight and about half of women have a height of $<150 \mathrm{~cm}^{(13)}$.

Adequate nutrition is an essential foundation for the health of individuals and populations. Underweight and stunting in women are not only associated with their poor health status but also that of their offspring, as widely evidenced by numerous studies on maternal nutrition and fetal and child health outcomes. Past research has solidified the relationship of maternal undernutrition (low BMI, stunting) with maternal health conditions such as chronic energy deficiency of mothers, caesarean delivery, preeclampsia, anaemia, loss of productivity and mental health, as well as adverse pregnancy outcomes ${ }^{(14-19)}$. Overweight and obese women are also predisposed to a wide range of health problems ${ }^{(20)}$, particularly an increased risk of acquiring hypertension, diabetes ${ }^{(21-23)}$, CVD and stroke ${ }^{(23)}$.

Undernutrition in women has been attributed to a multitude of factors, including upstream variables such as community-level WASH (water, sanitation and hygiene) practices $^{(24,25)}$, food stability status ${ }^{(26)}$, as well as household- and individual-level factors such as land ownership, household income and wealth, women's education level, age at first marriage, age at first delivery, multiparity and short birth interval ${ }^{(7,22,27-31)}$.

Robust estimates of levels and identification of determinants of nutritional status of women in resource-limited settings are important for targeting services and initiation of risk-specific interventions. Using data from a population-based cohort of non-pregnant women of childbearing age in a rural district of Bangladesh, we present the levels and correlates of nutritional status of rural Bangladeshi women.

\section{Methods}

\section{Study population}

The study was conducted in a rural field site in Sylhet District of north-eastern Bangladesh. The field site was established by the Projahnmo Study Group, a research partnership of Johns Hopkins University, USA, with the Bangladesh Ministry of Health and Family Welfare (MOHFW) and a number of Bangladeshi nongovernmental organizations (NGO). The site was established in 2001 to conduct clinical-epidemiological studies and intervention trials to contribute to improvements of maternal, newborn and child health ${ }^{(32,33)}$. The field site covers a population of about 500000 with about 60000 married women of childbearing age, aged 15-49 years, and an annual birth cohort of about 13000. Most of the population in this agrarian community is poor, with low levels of education, and more than a third of the men and women have no formal schooling.

The site has substantial infrastructure including: a census; a GPS (Global Positioning System)-based map; an updated population database maintained through home visits by locally recruited community health workers every two months; and data on background characteristics of the entire population which are updated periodically. In addition, study-specific data are collected as needed. Each woman has a current identification number for locating the woman and a permanent identification number allowing longitudinal linkages. An updated linked database is maintained to provide the sampling frame for current and future studies and to provide investigators the ability to link data on the same person across different studies for additional secondary analyses or study proposals. The present study was conducted in one part of the study area in a geographically contiguous population of about 100000 .

\section{Data sources}

We used the following data sources: (i) the census of the study area initially conducted in 2002 and continually updated. The census database provides information on woman's age, education, her husband's education and family size; (ii) data on household socio-economic status collected alongside the census and updated every 3 years. These data were collected using a standardized data collection form. The household socio-economic data include information on materials used to build the house, toilet facility, sources of drinking-water and household possessions; (iii) community (village)-level data including presence of a primary health-care centre operated by either the MOHFW or an NGO, collected along with the socioeconomic status data; (iv) data on time required to reach the sub-district hospital from the centre of each village calculated from a GIS (Geographic Information System) database; and (v) pre-pregnancy anthropometric data of married women of reproductive age collected at baseline of a cluster randomized trial designed to evaluate the impact of screening and treatment of pregnant women for bacterial vaginosis and urinary tract infection on preterm birth rate, known as the Maternal Infection Screening and Treatment (MIST) study ${ }^{(34)}$. Anthropometric data included weight and height and were measured by trained community health workers during 2010 and 2011. Weight was measured using a portable UNICEF Redline scale within the nearest $100 \mathrm{~g}$ and height was measured within the nearest $0 \cdot 1 \mathrm{~cm}$ using a locally constructed portable height stadiometer. Weighing scales were calibrated daily using known weights. 


\section{Data management}

We collected anthropometric data of 14731 women. To restrict the analysis to non-pregnant women of childbearing age, those who were pregnant during anthropometric measurement ( $n$ 908) and those below 15 years or over 49 years of age $(n$ 347) were excluded. A further 246 observations with implausible weight and height values (i.e. outliers) were excluded from the analysis. The hot deck method was used to impute values for missing data for the following variables: parity ( $n$ 3, $0.02 \%$ ); woman's education ( $n$ 488, 3.7\%); husband's age ( $n 636,4.8 \%)$; and household size ( $n 3,0.02 \%)$. In this procedure, other observations of the sample that have analogous characteristics were used to generate the missing values ${ }^{(35)}$. The final analytic file contained 13230 observations.

We categorized households according to their economic conditions by creating wealth scores based on house construction materials and household assets using principal component analysis and dividing them into quintiles. We calculated BMI from weight and height, which is defined as the ratio of weight in kilograms to the square of height in metres. We created a variable 'community-level food availability' as a proxy measure for community-level food shortages by dividing the calendar year into pre-harvest and post-harvest seasons. JulyDecember were considered as pre-harvest with presumed inadequate food availability and January-June were considered as post-harvest with presumed adequate food availability.

\section{Data analysis}

Women were categorized according to their BMI and height. They were classified into: underweight $\left(<18.5 \mathrm{~kg} / \mathrm{m}^{2}\right)$, normal $\left(18.5-24.9 \mathrm{~kg} / \mathrm{m}^{2}\right)$ and overweight/obese $\left(\geq 25.0 \mathrm{~kg} / \mathrm{m}^{2}\right)$ using BMI; and moderate to severely stunted $(<150 \mathrm{~cm})$, mildly stunted $(150-154 \mathrm{~cm})$ and normal $(\geq 155 \mathrm{~cm})$ using height. Bivariate and multivariate analyses were performed to measure the association between the two outcome variables (BMI, height) and selected individual, household sociodemographic and community-level characteristics. The association between two categorical variables was determined using the $\chi^{2}$ test. Results with a $P$ value of $<0.05$ were considered statistically significant. Two multinomial logistic regression models were fitted to identify risk factors for underweight and overweight/obese status using normal weight as reference category and adjusting for other covariates associated significantly at $P<0.05$ in bivariate analyses. Model 1 examined the association of individual and household factors, and model 2 additionally examined the effect of community variables. The models provided estimated relative risk ratios (RRR) and 95\% CI. Similar multinomial logistic regression models were fitted to examine risk factors for moderate to severe stunting $(<150 \mathrm{~cm})$ and mild stunting $(150-<155 \mathrm{~cm})$ using normal height $(\geq 155 \mathrm{~cm})$ as reference category. Analyses were conducted in the statistical software package Stata version 14 .

We obtained ethical approval for collection of data from the Johns Hopkins University Institutional Review Board and the International Centre for Diarrhoeal Disease Research, Bangladesh (icddr,b) Ethical Review Committee.

\section{Results}

Table 1 shows the frequency distribution including 95\% CI and the mean and SD of weight, height and BMI of the analytic cohort ( $n$ 13230). The mean weight, height and BMI were 44.8 (SD 8.0) kg, 149.9 (SD 5.6) $\mathrm{cm}$ and 19.9 (SD $3.3) \mathrm{kg} / \mathrm{m}^{2}$, respectively. The distribution of the study women across height categories shows that $16.5 \%$ of the women were severely stunted $(<145 \mathrm{~cm}), 32 \cdot 1 \%$ were moderately stunted and another $34.3 \%$ were mildly stunted. The distribution of the women across BMI categories shows that $55.8 \%$ of the women had normal weight, $37 \cdot 0 \%$ were underweight and $7 \cdot 2 \%$ were overweight/ obese (Table 1).

The distribution of the three categories of BMI of women was significantly associated with all variables examined including community-level food availability (Table 2). Underweight rate was $38.7 \%$ during the preharvest season when food availability is low and $35.9 \%$ in the post-harvest season $(P<0 \cdot 01$; Table 2$)$.

In the multivariable multinomial regression analyses, when groups with underweight and normal weight were compared in model 1, age, women's education, household socio-economic status and remittance were statistically

Table 1 Distribution of weight, height and BMI among the cohort of non-pregnant women of childbearing age $(n$ 13230) in rural Bangladesh

\begin{tabular}{|c|c|c|c|}
\hline Variable & $n$ & $\%$ & $95 \% \mathrm{Cl}$ \\
\hline \multicolumn{4}{|l|}{ Weight (kg) } \\
\hline$<35.0$ & 1012 & $7 \cdot 7$ & $7 \cdot 2,8 \cdot 1$ \\
\hline $35 \cdot 0-39.9$ & 2780 & $21 \cdot 0$ & $20 \cdot 3,21 \cdot 7$ \\
\hline $40 \cdot 0-44 \cdot 9$ & 3634 & $27 \cdot 5$ & $26 \cdot 7,28 \cdot 2$ \\
\hline $45 \cdot 0-49 \cdot 9$ & 2754 & $20 \cdot 8$ & $20 \cdot 1,21.5$ \\
\hline$\geq 50.0$ & 3050 & $23 \cdot 1$ & $22 \cdot 3,23 \cdot 8$ \\
\hline Mean & 44.8 & - & - \\
\hline SD & $8 \cdot 0$ & - & - \\
\hline \multicolumn{4}{|l|}{ Height $(\mathrm{cm})$} \\
\hline$<145.0$ & 2183 & 16.5 & $15 \cdot 9,17 \cdot 1$ \\
\hline $145 \cdot 0-149 \cdot 9$ & 4241 & $32 \cdot 1$ & $31 \cdot 3,32 \cdot 9$ \\
\hline $150 \cdot 0-154 \cdot 9$ & 4536 & 34.3 & $33.5,35 \cdot 1$ \\
\hline$\geq 155 \cdot 0$ & 2270 & $17 \cdot 2$ & $16 \cdot 5,17 \cdot 8$ \\
\hline Mean & 149.9 & - & - \\
\hline SD & $5 \cdot 6$ & - & - \\
\hline \multicolumn{4}{|l|}{ BMI $\left(\mathrm{kg} / \mathrm{m}^{2}\right)$} \\
\hline Underweight ( $\leq 18.5)$ & 4895 & $37 \cdot 0$ & $36 \cdot 2,37 \cdot 8$ \\
\hline Normal (18.6-24.9) & 7385 & $55 \cdot 8$ & $55 \cdot 0,56 \cdot 6$ \\
\hline Overweight (25.0-29.9) & 840 & $6 \cdot 4$ & $5 \cdot 9,6 \cdot 8$ \\
\hline Obese $(\geq 30 \cdot 0)$ & 110 & $0 \cdot 8$ & $0.7,1.0$ \\
\hline Mean & $19 \cdot 9$ & - & - \\
\hline SD & 3.3 & - & - \\
\hline
\end{tabular}


Table 2 Distribution of BMI categories by selected sociodemographic and community characteristics of the cohort of non-pregnant women of childbearing age $(n$ 13230) in rural Bangladesh

\begin{tabular}{|c|c|c|c|c|c|c|c|c|}
\hline \multirow[b]{2}{*}{ Characteristic } & \multirow[b]{2}{*}{$n$} & \multicolumn{2}{|c|}{$\begin{array}{l}\text { Underweight } \\
\left(\mathrm{BMI}<18.5 \mathrm{~kg} / \mathrm{m}^{2}\right)\end{array}$} & \multicolumn{2}{|c|}{$\begin{array}{c}\text { Normal weight } \\
\left(\mathrm{BMI}=18.5-24.9 \mathrm{~kg} / \mathrm{m}^{2}\right)\end{array}$} & \multicolumn{2}{|c|}{$\begin{array}{l}\text { Overweight/obese } \\
\left(\mathrm{BMI} \geq 25.0 \mathrm{~kg} / \mathrm{m}^{2}\right)\end{array}$} & \multirow[b]{2}{*}{$P$ value } \\
\hline & & $n$ & $\%$ & $n$ & $\%$ & $n$ & $\%$ & \\
\hline \multicolumn{9}{|l|}{ Individual } \\
\hline \multicolumn{9}{|l|}{ Age } \\
\hline$\leq 19$ years & 97 & 38 & $39 \cdot 2$ & 55 & $56 \cdot 7$ & 4 & $4 \cdot 1$ & $<0.001$ \\
\hline 20-24 years & 1702 & 589 & 34.6 & 1040 & 61.0 & 73 & $4 \cdot 3$ & \\
\hline $25-29$ years & 2162 & 693 & $32 \cdot 0$ & 1311 & $60 \cdot 6$ & 158 & $7 \cdot 3$ & \\
\hline 30-34 years & 3129 & 1111 & 35.5 & 1784 & $57 \cdot 0$ & 234 & 7.5 & \\
\hline$\geq 35$ years & 6140 & 2464 & $40 \cdot 1$ & 3195 & $52 \cdot 0$ & 481 & $7 \cdot 8$ & \\
\hline \multicolumn{9}{|l|}{ Parity } \\
\hline 0 & 940 & 335 & 35.6 & 520 & $55 \cdot 3$ & 85 & $9 \cdot 0$ & $<0.001$ \\
\hline $1-2$ & 4790 & 1660 & 34.7 & 2769 & $57 \cdot 8$ & 361 & 7.5 & \\
\hline $3-4$ & 4266 & 1573 & $36 \cdot 9$ & 2363 & 55.4 & 330 & 7.7 & \\
\hline $5-6$ & 2245 & 913 & $40 \cdot 6$ & 1196 & $53 \cdot 3$ & 136 & $6 \cdot 0$ & \\
\hline$\geq 7$ & 989 & 414 & 41.9 & 537 & $54 \cdot 3$ & 38 & 3.8 & \\
\hline \multicolumn{9}{|l|}{ Education } \\
\hline No education & 3461 & 1608 & 46.5 & 1745 & $50 \cdot 4$ & 108 & $3 \cdot 1$ & $<0.001$ \\
\hline $1-5$ years & 5755 & 2122 & 36.9 & 3252 & $56 \cdot 5$ & 381 & 6.6 & \\
\hline $6-10$ years & 3791 & 1116 & $29 \cdot 4$ & 2248 & $59 \cdot 3$ & 427 & $11 \cdot 3$ & \\
\hline$\geq 11$ years & 223 & 49 & $22 \cdot 0$ & 140 & $62 \cdot 8$ & 34 & $15 \cdot 2$ & \\
\hline \multicolumn{9}{|l|}{ NGO membership } \\
\hline Yes & 1564 & 663 & $42 \cdot 4$ & 831 & $53 \cdot 1$ & 70 & 4.5 & $<0.001$ \\
\hline No & 11666 & 4232 & $36 \cdot 3$ & 6554 & $56 \cdot 2$ & 880 & 7.5 & \\
\hline \multicolumn{9}{|l|}{ Household } \\
\hline Wealth quintile & & & & & & & & \\
\hline Lowest quintile & 3074 & 1497 & $48 \cdot 7$ & 1550 & $48 \cdot 8$ & 77 & 2.5 & $<0.001$ \\
\hline Second lowest quintile & 2239 & 952 & $42 \cdot 5$ & 1205 & $53 \cdot 8$ & 82 & $3 \cdot 7$ & \\
\hline Middle quintile & 2172 & 862 & $39 \cdot 7$ & 1183 & 54.5 & 127 & $5 \cdot 8$ & \\
\hline Second highest quintile & 2539 & 839 & $33 \cdot 0$ & 1508 & $59 \cdot 4$ & 192 & $7 \cdot 6$ & \\
\hline Highest quintile & 3206 & 745 & $23 \cdot 2$ & 1989 & $62 \cdot 0$ & 472 & $14 \cdot 7$ & \\
\hline Husband's education & & & & & & & & \\
\hline No education & 3485 & 1616 & $46 \cdot 4$ & 1759 & $50 \cdot 5$ & 110 & $3 \cdot 2$ & $<0.001$ \\
\hline $1-5$ years & 5401 & 2027 & 37.5 & 3060 & $56 \cdot 7$ & 314 & $5 \cdot 8$ & \\
\hline $6-10$ years & 3470 & 1056 & 30.4 & 2044 & $58 \cdot 9$ & 370 & $10 \cdot 7$ & \\
\hline$\geq 11$ years & 874 & 196 & $22 \cdot 4$ & 522 & $59 \cdot 7$ & 156 & $17 \cdot 8$ & \\
\hline Family size & & & & & & & & \\
\hline $1-4$ & 2241 & 842 & $37 \cdot 6$ & 1229 & $54 \cdot 8$ & 170 & $7 \cdot 6$ & $<0.05$ \\
\hline $5-6$ & 3451 & 1284 & $37 \cdot 2$ & 1918 & $55 \cdot 6$ & 249 & $7 \cdot 2$ & \\
\hline $7-8$ & 2972 & 1166 & $39 \cdot 2$ & 1603 & 53.9 & 203 & $6 \cdot 8$ & \\
\hline$\geq 9$ & 4566 & 1603 & $35 \cdot 1$ & 2635 & $57 \cdot 7$ & 328 & $7 \cdot 2$ & \\
\hline Religion & & & & & & & & \\
\hline Muslim & 12332 & 4499 & $36 \cdot 5$ & 6910 & $56 \cdot 0$ & 923 & 7.5 & $<0.001$ \\
\hline Other & 898 & 396 & $44 \cdot 1$ & 475 & 52.9 & 27 & 3.0 & \\
\hline Type of latrine & & & & & & & & \\
\hline Improved† & 10721 & 3863 & $36 \cdot 0$ & 6019 & $56 \cdot 1$ & 839 & $7 \cdot 8$ & $<0.001$ \\
\hline Non-improved $\ddagger$ & 2509 & 1032 & $41 \cdot 1$ & 1366 & 54.4 & 111 & 4.4 & \\
\hline Source of drinking-water & & & & & & & & \\
\hline Improved§ & 6185 & 2228 & $36 \cdot 0$ & 3421 & $55 \cdot 3$ & 536 & 8.7 & $<0.001$ \\
\hline Non-improved $\|$ & 7045 & 2667 & $37 \cdot 9$ & 3964 & $56 \cdot 3$ & 414 & $5 \cdot 9$ & \\
\hline Type of cooking fuel & & & & & & & & \\
\hline Improved & 71 & 10 & $14 \cdot 1$ & 48 & $67 \cdot 6$ & 13 & $18 \cdot 3$ & $<0.0001$ \\
\hline Non-improved†† & 13159 & 4885 & $37 \cdot 1$ & 7337 & $55 \cdot 8$ & 937 & $7 \cdot 1$ & \\
\hline Remittance & & & & & & & & \\
\hline Yes & 2802 & 751 & $26 \cdot 8$ & 1738 & $62 \cdot 0$ & 313 & $11 \cdot 2$ & $<0.001$ \\
\hline No & 10428 & 4144 & 39.7 & 5647 & $54 \cdot 1$ & 637 & $6 \cdot \overline{1}$ & \\
\hline Community & & & & & & & & \\
\hline Food availability & & & & & & & & \\
\hline Pre-harvestł & 5173 & 2004 & $38 \cdot 7$ & 2780 & $53 \cdot 7$ & 389 & 7.5 & $<0.01$ \\
\hline Post-harvest§§ & 8057 & 2891 & 35.9 & 4605 & $57 \cdot 2$ & 561 & $7 \cdot 0$ & \\
\hline Time to upazila headquart & & & & & & & & \\
\hline$<30 \min$ & 1360 & 450 & $33 \cdot 1$ & 831 & $61 \cdot 1$ & 79 & $5 \cdot 8$ & $<0.001$ \\
\hline $30-44 \min$ & 7204 & 2711 & $37 \cdot 6$ & 3942 & $54 \cdot 7$ & 551 & $7 \cdot 7$ & \\
\hline$\geq 45 \mathrm{~min}$ & 4666 & 1734 & $37 \cdot 2$ & 2612 & $56 \cdot 0$ & 320 & 6.9 & \\
\hline Availability of MOHFW or & & & & & & & & \\
\hline Yes & 785 & 255 & 32.5 & 461 & $58 \cdot 7$ & 69 & $8 \cdot 8$ & 0.01 \\
\hline No & 12445 & 4640 & $37 \cdot 3$ & 6924 & $55 \cdot 6$ & 881 & $7 \cdot 1$ & \\
\hline
\end{tabular}

NGO, non-governmental organization; MOHFW, Ministry of Health and Family Welfare.

†Improved latrine included all flushed and pit latrines with slab.

$\ddagger$ Non-improved latrine included pit latrine without slab, hanging latrine, dry latrine and no latrine/bush/field.

§Improved sources of drinking-water included water from pipe/tap, tube well and tank.

|| Non-improved sources of drinking-water included water from dug well, spring, rain and river/dam/lake/pond/stream/canal.

IImproved cooking fuel included cooking by electric, liquefied petroleum gas and kerosene.

††Non-improved cooking fuel included cooking by using wood, charcoal, straw/shrubs/grass, agricultural crop and animal dung

łłPre-harvest: period between July and December of a year.

$\S \S$ Post-harvest: period between January and June of a year. 
significantly associated with undernutrition. Compared with women aged 25-29 years, risk of undernutrition was higher in women older than 35 years $(\mathrm{RRR}=1 \cdot 34$; $95 \% \mathrm{CI}$ $1 \cdot 19,1.51$ ). The risk of undernutrition was inversely related to women's education, household wealth and remittance (Table 3). In model 2 of underweight $v$. normal weight comparison, risk of underweight was significantly lower in the post-harvest season ( $\mathrm{RRR}=0 \cdot 82 ; 95 \% \mathrm{CI} 0 \cdot 76$, $0 \cdot 89$ ) with presumed higher food availability at the community level. When groups with normal weight and overweight/obesity were compared in model 1, compared with women aged 25-29 years, the risk of overweight was significantly higher in women older than 30 years of age (30-34 years: $R R R=1 \cdot 33 ; 95 \%$ CI 1.06, 1.67; $\geq 35$ years of age: $\mathrm{RRR}=1 \cdot 88 ; 95 \%$ CI $1.52,2 \cdot 33$ ), women who had any education, women who belonged to households with higher wealth, and women having an improved latrine and an improved source of drinking-water (Table 3).

In bivariate analysis, women's education, husband's education, religion, NGO membership, household wealth, household's access to improved toilet, improved drinkingwater, remittance, time to go to the sub-district (upazila) headquarters, and availability of an MOHFW or NGO clinic in the village were significantly associated with height categories (Table 4). Women with secondary education were less likely to be moderate to severely stunted in both model 1 (RRR $=0.75 ; 95 \%$ CI 0.65, 0.86) and model $2(\mathrm{RRR}=0 \cdot 76 ; 95 \%$ CI $0 \cdot 67,0 \cdot 87)$. Women who belonged to the highest wealth quintiles were significantly less likely to be moderate to severely stunted in model 1 (RRR $=0.67$; $95 \%$ CI $0.58,0.79)$ and model $2(\mathrm{RRR}=0.66 ; 95 \%$ CI 0.57 , 0.77 ; Table 5). Women in the second highest wealth quintiles were also significantly less likely to be moderate to severely stunted in model 1 (RRR $=0.77 ; 95 \%$ CI 0.66 , $0.91)$ and model 2 (RRR $=0.77 ; 95 \%$ CI $0.65,0.90$; Table 5). Women other than Muslim were at a significantly higher risk of being moderate to severely stunted as well as mildly stunted in both models (Table 5).

\section{Discussion}

In this population-based cohort of women of childbearing age, underweight and moderate to severe stunting rates were high at 37.0 and $48.6 \%$, respectively. About $17 \%$ of the women were severely stunted (height $<145 \mathrm{~cm}$ ) and about another a third were moderately stunted (height $=$ $145-<150 \mathrm{~cm}$ ). About $7 \%$ of the women were overweight or obese.

Underweight status was associated with individual-level factors such as age; older women were experiencing the highest risk of being underweight. Several other individual and household factors including educational attainment of women, household wealth and remittance were inversely associated with underweight status. The associations remained same after addition of community-level factors.
Compared with women living in villages within 30 min travel distance from the sub-district headquarters, women residing in villages with a travel time of more than $30 \mathrm{~min}$ were more likely to be underweight. The risk of underweight among women of childbearing age was lower in the post-harvest season and in villages with an MOHFW or NGO health clinic. Maternal overweight/ obesity was found to be positively associated with individual-level factors including increasing age, higher parity and higher educational attainment; and householdlevel factors including higher household wealth, improved latrine and improved source of drinking-water. These associations remained unchanged after inclusion of community-level variables. Our findings highlight the importance of household- and community-level factors in addition to individual-level factors on likelihood of women to be underweight as well as overweight/obese.

The present study documented a high prevalence of underweight among women of childbearing age in Bangladesh. This is similar to earlier findings from Bangladesh $^{(27,36)}$ and India ${ }^{(37,38)}$. Using Bangladesh Demographic and Health Survey (BDHS) 2011 data of married Bangladeshi women, Islam et al. ${ }^{(27)}$ reported an underweight rate of $32 \cdot 1 \%$. Using the Indian National Family Health Survey (NFHS) data collected across twenty-one states of India during 1998-1999 and 20052006, Sengupta et al. ${ }^{(37)}$ reported that almost one out of three Indian ever-married women was underweight. A large community-based study in India reported similar findings, where $31.2 \%$ of women were underweight and $12.0 \%$ of women were overweight or obese ${ }^{(38)}$. However, the underweight rate in Bangladesh as a whole is declining; the proportion of women who are underweight $\left(\mathrm{BMI}<18.5 \mathrm{~kg} / \mathrm{m}^{2}\right)$ has declined from 34.0 to $19.0 \%$ between 2004 and $2014^{(13)}$.

Our study also documented a low to moderate prevalence of overweight/obesity, similar to several studies conducted in Bangladesh and India ${ }^{(36,38)}$. The overweight/ obesity rate we observed was lower compared with some other studies conducted in Bangladesh ${ }^{(7,13)}$. BDHS 2014 data revealed that overweight or obesity (BMI $\geq$ $25 \cdot 0 \mathrm{~kg} / \mathrm{m}^{2}$ ) among ever-married women aged 15-49 years in Bangladesh has been increasing over the past decade, from $9 \%$ in 2004 to $24 \%$ in $2014^{(13)}$. The present study was conducted in a rural area in Sylhet Division, a division with the lowest prevalence of overweight (15.2\%) among the eight divisions of Bangladesh ${ }^{(13)}$. The lower rate we observed may be due to differences in population $^{(22,28)}$. It has been shown that in Bangladesh the underweight rate in women is higher among rural than urban residents (21 and 12\%, respectively), whereas urban women are twice more likely to be overweight or obese compared with rural women (36 and 19\%, respectively $)^{(7,13)}$. Therefore, the actual burden of overweight or obesity in Bangladesh is much higher than what we have observed and, seemingly, Bangladesh is in an 
Table 3 Multinomial logistic regression of selected sociodemographic and community characteristics associated with low and high BMI among the cohort of non-pregnant women of childbearing age $(n$ 13230) in rural Bangladesh

\begin{tabular}{|c|c|c|c|c|c|c|c|c|}
\hline \multirow[b]{3}{*}{ Characteristic } & \multicolumn{4}{|c|}{ Underweight $v$. normal weight } & \multicolumn{4}{|c|}{ Overweight/obese $v$. normal weight } \\
\hline & \multicolumn{2}{|c|}{ Model 1} & \multicolumn{2}{|c|}{ Model 2} & \multicolumn{2}{|c|}{ Model 1} & \multicolumn{2}{|c|}{ Model 2} \\
\hline & RRR & $95 \% \mathrm{Cl}$ & RRR & $95 \% \mathrm{Cl}$ & RRR & $95 \% \mathrm{Cl}$ & RRR & $95 \% \mathrm{Cl}$ \\
\hline \multicolumn{9}{|l|}{ Individual } \\
\hline \multicolumn{9}{|l|}{ Age } \\
\hline$\leq 19$ years & 1.42 & $0.92,2.19$ & 1.41 & $0.92,2.18$ & 0.49 & $0.17,1.39$ & 0.49 & $0.17,1.39$ \\
\hline 20-24 years & $1 \cdot 11$ & $0.96,1.28$ & $1 \cdot 11$ & $0.96,1.28$ & 0.52 & $0.39,0.70^{\star \star \star}$ & 0.53 & $0.39,0.71^{\star \star *}$ \\
\hline 25-29 years & & Ref. & & Ref. & & Ref. & & Ref. \\
\hline 30-34 years & $1 \cdot 10$ & $0.97,1.25$ & $1 \cdot 10$ & $0.97,1.25$ & $1 \cdot 33$ & $1.07,1.67^{*}$ & $1 \cdot 33$ & $1.07,1.67^{*}$ \\
\hline$\geq 35$ years & $1 \cdot 34$ & $1.19,1.51^{\star \star \star}$ & $1 \cdot 35$ & $1 \cdot 20,1.52^{\star \star \star}$ & $1 \cdot 88$ & $1 \cdot 52,2 \cdot 33^{\star \star \star}$ & 1.88 & $1 \cdot 51,2 \cdot 32^{\star \star \star}$ \\
\hline \multicolumn{9}{|l|}{ Parity } \\
\hline 0 & & Ref. & & Ref. & & Ref. & & Ref. \\
\hline $1-2$ & 0.96 & $0.83,1.12$ & 0.97 & $0.83,1.13$ & 0.82 & $0.63,1.07$ & 0.83 & $0.63,1.07$ \\
\hline $3-4$ & 0.91 & $0.78,1.07$ & 0.91 & $0.78,1.07$ & 0.78 & $0.60,1.03$ & 0.79 & $0.60,1.03$ \\
\hline $5-6$ & 0.92 & $0.77,1.10$ & 0.92 & $0.77,1.10$ & 0.66 & $0.48,0.90^{\star \star}$ & 0.66 & $0.48,0.90^{\star *}$ \\
\hline$\geq 7$ & 0.87 & $0.71,1.07$ & 0.87 & $0.71,1.07$ & 0.47 & $0.30,0.71^{\star \star \star}$ & 0.47 & $0.31,0.71^{\star * *}$ \\
\hline \multicolumn{9}{|l|}{ Education } \\
\hline No education & & Ref. & & Ref. & & Ref. & & Ref. \\
\hline $1-5$ years & 0.81 & $0.74,0.89^{* \star *}$ & 0.81 & $0.74,0.89^{* \star \star}$ & $1 \cdot 80$ & $1 \cdot 43,2 \cdot 26^{\star \star \star}$ & 1.79 & $1 \cdot 43,2 \cdot 25^{\star \star *}$ \\
\hline $6-10$ years & 0.73 & $0.65,0.82^{\star \star \star}$ & 0.72 & $0.64,0.81^{\star \star *}$ & 2.72 & $2 \cdot 14,3 \cdot 46^{\star \star \star}$ & $2 \cdot 70$ & $2 \cdot 12,3 \cdot 43^{\star * *}$ \\
\hline$\geq 11$ years & 0.60 & $0.42,0.84^{\star *}$ & 0.59 & $0.42,0.83^{\star \star}$ & 3.02 & $1.93,4.72^{\star \star \star}$ & 2.97 & $1.90,4 \cdot 64^{\star * *}$ \\
\hline \multicolumn{9}{|l|}{ NGO membership } \\
\hline No & & Ref. & & Ref. & & Ref. & & Ref. \\
\hline Yes & 1.05 & $0.94,1.18$ & 1.08 & $0.97,1.21$ & 0.89 & $0.68,1.15$ & 0.90 & $0.69,1.17$ \\
\hline \multicolumn{9}{|l|}{ Household } \\
\hline \multicolumn{9}{|l|}{ Wealth quintile } \\
\hline Lowest quintile & & Ref. & & Ref. & & Ref. & & Ref. \\
\hline Second lowest quintile & 0.84 & $0.75,0.94^{* *}$ & 0.84 & $0.75,0.94^{\star *}$ & $1 \cdot 11$ & $0.80,1.53$ & $1 \cdot 12$ & $0.81,1.55$ \\
\hline Middle quintile & 0.79 & $0.71,0.89^{\star \star \star}$ & 0.79 & $0.71,0.89^{* \star *}$ & 1.63 & $1 \cdot 21,2 \cdot 20^{\star \star}$ & 1.64 & $1 \cdot 22,2 \cdot 21^{\star *}$ \\
\hline Second highest quintile & 0.64 & $0.57,0.72^{* \star *}$ & 0.63 & $0.56,0.71^{\star \star *}$ & 1.71 & $1 \cdot 29,2 \cdot 27^{\star \star \star}$ & 1.71 & $1 \cdot 29,2 \cdot 27^{\star \star *}$ \\
\hline Highest quintile & 0.45 & $0.40,0.51^{\star * *}$ & 0.46 & $0.40,0.53^{\star * *}$ & 3.09 & $2 \cdot 36,4 \cdot 04^{\star \star \star}$ & 3.12 & $2 \cdot 39,4 \cdot 08^{\star \star *}$ \\
\hline Family size & & & & & & & & \\
\hline $1-4$ & & Ref. & & Ref. & & Ref. & & Ref. \\
\hline $5-6$ & 0.95 & $0.84,1.07$ & 0.95 & $0.84,1.07$ & 0.90 & $0.72,1.12$ & 0.90 & $0.72,1.12$ \\
\hline $7-8$ & 1.00 & $0.89,1.14$ & 1.01 & $0.89,1.14$ & 0.95 & $0.75,1.20$ & 0.96 & $0.76,1.21$ \\
\hline$\geq 9$ & 0.96 & $0.85,1.07$ & 0.96 & $0.85,1.07$ & 0.85 & $0.69,1.04$ & 0.85 & $0.69,1.05$ \\
\hline Religion & & & & & & & & \\
\hline Muslim & & Ref. & & Ref. & & Ref. & & Ref. \\
\hline Other & 1.07 & $0.93,1.24$ & $1 \cdot 13$ & $0.97,1.31$ & 0.48 & $0.32,0.72^{\star \star \star}$ & 0.52 & $0.34,0.78^{\star *}$ \\
\hline Type of latrine & & & & & & & & \\
\hline Improved† & 0.94 & $0.85,1.04$ & 0.93 & $0.84,1.03$ & 1.48 & $1 \cdot 17,1.87^{\star \star}$ & 1.48 & $1 \cdot 17,1 \cdot 87^{\star *}$ \\
\hline Non-improved $\ddagger$ & & Ref. & & Ref. & & Ref. & & Ref. \\
\hline Source of drinking-water & & & & & & & & \\
\hline Improved§ & 1.02 & $0.94,1.11$ & 1.02 & $0.94,1.10$ & $1 \cdot 33$ & $1 \cdot 15,1.55^{\star \star \star}$ & $1 \cdot 33$ & $1 \cdot 14,1 \cdot 54^{\star * *}$ \\
\hline Non-improved & & Ref. & & Ref. & & Ref. & & Ref. \\
\hline Type of cooking fuel & & & & & & & & \\
\hline Improved & 0.53 & $0.27,1.07$ & 0.53 & $0.26,1.06$ & $1 \cdot 22$ & $0.65,2.30$ & $1 \cdot 25$ & $0.66,2.36$ \\
\hline Non-improved†† & & Ref. & & Ref. & & Ref. & & Ref. \\
\hline Remittance & & & & & & & & \\
\hline No & & Ref. & & Ref. & & Ref. & & Ref. \\
\hline Yes & 0.78 & $0.70,0.86^{\star \star \star}$ & 0.78 & $0.70,0.86^{\star \star \star}$ & 1.04 & $0.88,1.22$ & 1.04 & $0.88,1.22$ \\
\hline Community & & & & & & & & \\
\hline Food availability & & & & & & & & \\
\hline Pre-harvestł‡ & & & & Ref. & & & & Ref. \\
\hline Post-harvest§§ & & & 0.82 & $0.76,0.89^{\star \star \star}$ & & & 0.92 & $0.80,1.06$ \\
\hline Time to upazila headquart & & & & & & & & \\
\hline$<30 \min$ & & & & Ref. & & & & Ref. \\
\hline $30-44 \min$ & & & $1 \cdot 30$ & $1.14,1.47^{\star \star \star}$ & & & $1 \cdot 34$ & $1 \cdot 04,1 \cdot 73^{*}$ \\
\hline$\geq 45 \mathrm{~min}$ & & & $1 \cdot 20$ & $1.04,1.37^{\star}$ & & & 1.34 & $1.02,1 \cdot 74^{\star}$ \\
\hline Availability of MOHFW or & & & & & & & & \\
\hline No & & & & Ref. & & & & Ref. \\
\hline Yes & & & 0.85 & $0.73,1.01$ & & & 1.02 & $0.77,1.33$ \\
\hline
\end{tabular}

RRR, relative risk ratio; NGO, non-governmental organization; MOHFW, Ministry of Health and Family Welfare; Ref., reference category. Model 1 examined the association of individual and household factors; model 2 additionally examined the effect of community variables. ${ }^{\star} P<0.05,{ }^{\star \star} P<0.01,{ }^{\star \star \star} P<0.001$.

†Improved latrine included all flushed and pit latrines with slab.

$\ddagger$ Non-improved latrine included pit latrine without slab, hanging latrine, dry latrine and no latrine/bush/field.

$\S$ Improved sources of drinking-water included water from pipe/tap, tube well and tank.

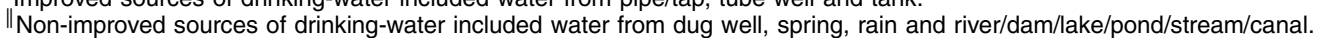

IImproved cooking fuel included cooking by electric, liquefied petroleum gas and kerosene.

${ }^{+\dagger}$ Non-improved cooking fuel included cooking by using wood, charcoal, straw/shrubs/grass, agricultural crop and animal dung.

¥¥Pre-harvest: period between July and December of a year.

$\S \S$ Post-harvest: period between January and June of a year. 
Table 4 Distribution of height by selected sociodemographic and community characteristics of the cohort of non-pregnant women of childbearing age ( $n$ 13230) in rural Bangladesh

\begin{tabular}{|c|c|c|c|c|c|c|c|c|}
\hline \multirow[b]{2}{*}{ Characteristics } & \multirow[b]{2}{*}{$n$} & \multicolumn{2}{|c|}{$\begin{array}{l}\text { Moderate to severe stunting } \\
\quad \text { (height }<150 \mathrm{~cm})\end{array}$} & \multicolumn{2}{|c|}{$\begin{array}{c}\text { Mild stunting } \\
\text { (height }=150-<155 \mathrm{~cm})\end{array}$} & \multicolumn{2}{|c|}{$\begin{array}{c}\text { Normal height } \\
\text { (height } \geq 155 \mathrm{~cm} \text { ) }\end{array}$} & \multirow[b]{2}{*}{$P$ value } \\
\hline & & $n$ & $\%$ & $n$ & $\%$ & $n$ & $\%$ & \\
\hline \multicolumn{9}{|l|}{ Individual } \\
\hline \multicolumn{9}{|l|}{ Age } \\
\hline$\leq 19$ years & 97 & 47 & 48.5 & 36 & $37 \cdot 1$ & 14 & 14.4 & 0.633 \\
\hline $20-24$ years & 1702 & 843 & 49.5 & 581 & 34.1 & 278 & $16 \cdot 3$ & \\
\hline $25-29$ years & 2162 & 1060 & 49.0 & 742 & 34.3 & 360 & $16 \cdot 7$ & \\
\hline $30-34$ years & 3129 & 1544 & 49.3 & 1067 & 34.1 & 518 & $16 \cdot 6$ & \\
\hline$\geq 35$ years & 6140 & 2930 & $47 \cdot 7$ & 2110 & 34.4 & 1100 & 17.9 & \\
\hline \multicolumn{9}{|l|}{ Parity } \\
\hline 0 & 940 & 435 & $46 \cdot 3$ & 348 & $37 \cdot 0$ & 157 & $16 \cdot 7$ & 0.519 \\
\hline $1-2$ & 4790 & 2288 & 47.8 & 1648 & 34.4 & 854 & $17 \cdot 8$ & \\
\hline $3-4$ & 4266 & 2105 & 49.3 & 1443 & 33.8 & 718 & $16 \cdot 8$ & \\
\hline $5-6$ & 2245 & 1111 & 49.5 & 762 & 33.9 & 372 & $16 \cdot 6$ & \\
\hline$\geq 7$ & 989 & 485 & $49 \cdot 0$ & 335 & 33.9 & 169 & $17 \cdot 1$ & \\
\hline \multicolumn{9}{|l|}{ Education } \\
\hline No education & 3461 & 1795 & 51.9 & 1129 & 32.6 & 537 & $15 \cdot 5$ & $<0.001$ \\
\hline $1-5$ years & 5755 & 2898 & 50.4 & 1923 & 33.4 & 934 & $16 \cdot 2$ & \\
\hline $6-10$ years & 3791 & 1635 & $43 \cdot 1$ & 1408 & $37 \cdot 1$ & 748 & $19 \cdot 7$ & \\
\hline$\geq 11$ years & 223 & 96 & $43 \cdot 1$ & 76 & 34.1 & 51 & 22.9 & \\
\hline \multicolumn{9}{|l|}{ NGO membership } \\
\hline Yes & 1564 & 846 & $54 \cdot 1$ & 491 & 31.4 & 227 & 14.5 & $<0.001$ \\
\hline No & 11666 & 5578 & $47 \cdot 8$ & 4045 & 34.7 & 2043 & 17.5 & \\
\hline \multicolumn{9}{|l|}{ Household } \\
\hline Wealth quintile & & & & & & & & \\
\hline Lowest quintile & 3074 & 1637 & $53 \cdot 3$ & 997 & 32.4 & 440 & $14 \cdot 3$ & $<0.001$ \\
\hline Second lowest quintile & 2239 & 1113 & 49.7 & 780 & 34.8 & 346 & 15.5 & \\
\hline Middle quintile & 2172 & 1123 & $51 \cdot 7$ & 699 & $32 \cdot 2$ & 350 & $16 \cdot 1$ & \\
\hline Second highest quintile & 2539 & 1164 & $45 \cdot 8$ & 900 & 35.5 & 475 & $18 \cdot 7$ & \\
\hline Highest quintile & 3206 & 1387 & 43.3 & 1160 & $36 \cdot 2$ & 659 & 20.6 & \\
\hline Husband's education & & & & & & & & \\
\hline No education & 3485 & 1807 & 51.9 & 1136 & 32.6 & 542 & $15 \cdot 6$ & $<0.001$ \\
\hline $1-5$ years & 5401 & 2759 & $51 \cdot 1$ & 1825 & 33.8 & 817 & $15 \cdot 1$ & \\
\hline $6-10$ years & 3470 & 1517 & 43.7 & 1250 & $36 \cdot 0$ & 703 & $20 \cdot 3$ & \\
\hline$\geq 11$ years & 874 & 341 & 39.0 & 325 & 37.2 & 208 & 23.8 & \\
\hline Family size & & & & & & & & \\
\hline $1-4$ & 2241 & 1087 & 48.5 & 775 & 34.6 & 379 & $16 \cdot 9$ & 0.414 \\
\hline $5-6$ & 3451 & 1712 & 49.6 & 1151 & 33.4 & 588 & $17 \cdot 0$ & \\
\hline $7-8$ & 2972 & 1460 & $49 \cdot 1$ & 1026 & 34.5 & 486 & $16 \cdot 4$ & \\
\hline$\geq 9$ & 4566 & 2165 & $47 \cdot 4$ & 1584 & 34.7 & 817 & 17.9 & \\
\hline Religion & & & & & & & & \\
\hline Muslim & 12332 & 5879 & $47 \cdot 7$ & 4267 & 34.6 & 2186 & $17 \cdot 7$ & $<0.001$ \\
\hline Other & 898 & 545 & 60.7 & 269 & 30.0 & 84 & 9.4 & \\
\hline Type of latrine & & & & & & & & \\
\hline Improved $\dagger$ & 10721 & 5146 & $48 \cdot 0$ & 3711 & 34.6 & 1864 & $17 \cdot 4$ & $<0.05$ \\
\hline Non-improved $\ddagger$ & 2509 & 1278 & 50.9 & 825 & 32.9 & 406 & $16 \cdot 2$ & \\
\hline Source of drinking-water & & & & & & & & \\
\hline Improved§ & 6185 & 2879 & $46 \cdot 6$ & 2164 & 35.0 & 1142 & 18.5 & $<0.001$ \\
\hline Non-improved $\|$ & 7045 & 3545 & $50 \cdot 3$ & 2372 & 33.7 & 1128 & $16 \cdot 0$ & \\
\hline Type of cooking fuel & & & & & & & & \\
\hline Improvedף & 71 & 34 & 47.9 & 20 & $28 \cdot 2$ & 17 & 23.9 & 0.259 \\
\hline Non-improvedt† & 13159 & 6390 & $48 \cdot 6$ & 4516 & $34 \cdot 3$ & 2253 & $17 \cdot 1$ & \\
\hline Remittance & & & & & & & & \\
\hline Yes & 2802 & 1279 & $45 \cdot 7$ & 1020 & $36 \cdot 4$ & 503 & $18 \cdot 0$ & $<0.01$ \\
\hline No & 10428 & 5145 & 49.3 & 3516 & 33.7 & 1767 & $16 \cdot 9$ & \\
\hline Community & & & & & & & & \\
\hline Food availability & & & & & & & & \\
\hline Pre-harvestł‡ & 5173 & 2539 & $49 \cdot 1$ & 1765 & 34.1 & 869 & $16 \cdot 8$ & 0.551 \\
\hline Post-harvest§§ & 8057 & 3885 & $48 \cdot 2$ & 2771 & 34.4 & 1401 & $17 \cdot 4$ & \\
\hline Time to upazila headquart & & & & & & & & \\
\hline$<30 \mathrm{~min}$ & 1360 & 756 & $55 \cdot 6$ & 454 & 33.4 & 150 & 11.0 & $<0.001$ \\
\hline $30-44 \mathrm{~min}$ & 7204 & 3328 & $46 \cdot 2$ & 2461 & 34.2 & 1415 & $19 \cdot 6$ & \\
\hline$\geq 45 \min$ & 4666 & 2340 & $50 \cdot 2$ & 1621 & 34.7 & 705 & $15 \cdot 1$ & \\
\hline Availability of MOHFW or & & & & & & & & \\
\hline Yes & 785 & 348 & 44.3 & 312 & 39.8 & 125 & $15 \cdot 9$ & 0.01 \\
\hline No & 12445 & 6076 & $48 \cdot 8$ & 4224 & 33.9 & 2145 & $17 \cdot 2$ & \\
\hline
\end{tabular}

NGO, non-governmental organization; MOHFW, Ministry of Health and Family Welfare.

†Improved latrine included all flushed and pit latrines with slab.

$\ddagger$ Non-improved latrine included pit latrine without slab, hanging latrine, dry latrine and no latrine/bush/field.

§Improved sources of drinking-water included water from pipe/tap, tube well and tank.

$\|$ Non-improved sources of drinking-water included water from dug well, spring, rain and river/dam/lake/pond/stream/canal.

IIImproved cooking fuel included cooking by electric, liquefied petroleum gas and kerosene.

t†Non-improved cooking fuel included cooking by using wood, charcoal, straw/shrubs/grass, agricultural crop and animal dung.

¥¥Pre-harvest: period between July and December of a year.

$\S \S$ Post-harvest: period between January and June of a year. 
Table 5 Multinomial regression of selected sociodemographic and community characteristics associated with height among the cohort of non-pregnant women of childbearing age $(n 13230)$ in rural Bangladesh

\begin{tabular}{|c|c|c|c|c|c|c|c|c|}
\hline \multirow[b]{3}{*}{ Characteristic } & \multicolumn{4}{|c|}{$\begin{array}{l}\text { Moderate to severe stunting } v \text {. normal height } \\
\text { (height }<150 \mathrm{~cm} v \text {. height } \geq 155 \mathrm{~cm} \text { ) }\end{array}$} & \multicolumn{4}{|c|}{$\begin{array}{l}\text { Mild stunting } v \text {. normal height } \\
\text { (height }=150-<155 \mathrm{~cm} v \text {. height } \geq 155 \mathrm{~cm} \text { ) }\end{array}$} \\
\hline & \multicolumn{2}{|c|}{ Model 1} & \multicolumn{2}{|c|}{ Model 2} & \multicolumn{2}{|r|}{ Model 1} & \multicolumn{2}{|r|}{ Model 2} \\
\hline & RRR & $95 \% \mathrm{Cl}$ & RRR & $95 \% \mathrm{Cl}$ & RRR & $95 \% \mathrm{Cl}$ & RRR & $95 \% \mathrm{Cl}$ \\
\hline \multicolumn{9}{|l|}{ Individual } \\
\hline \multicolumn{9}{|l|}{ Education } \\
\hline No education & & Ref. & & Ref. & & Ref. & & Ref. \\
\hline $1-5$ years & 0.97 & $0.86,1 \cdot 10$ & 0.98 & $0.86,1.11$ & 1.00 & $0.88,1.14$ & 1.00 & $0.88,1.14$ \\
\hline $6-10$ years & 0.75 & $0.65,0.86^{\star \star *}$ & 0.76 & $0.67,0.87^{* \star *}$ & 0.95 & $0.83,1.10$ & 0.96 & $0.83,1.11$ \\
\hline$\geq 11$ years & 0.71 & $0.49,1.02$ & 0.73 & $0.51,1.05$ & 0.79 & $0.54,1 \cdot 15$ & 0.80 & $0.55,1.17$ \\
\hline \multicolumn{9}{|l|}{ NGO membership } \\
\hline No & & Ref. & & Ref. & & Ref. & & Ref. \\
\hline Yes & 1.17 & $1.01,1.37$ & $1 \cdot 12$ & $0.96,1.32$ & 1.01 & $0.86,1.20$ & 0.98 & $0.83,1.16$ \\
\hline \multicolumn{9}{|l|}{ Household } \\
\hline \multicolumn{9}{|l|}{ Wealth quintile } \\
\hline Lowest quintile & & Ref. & & Ref. & & Ref. & & Ref. \\
\hline Second lowest quintile & 0.93 & $0.79,1.09$ & 0.93 & $0.79,1.09$ & 1.01 & $0.86,1.20$ & 1.02 & $0.86,1.21$ \\
\hline Middle quintile & 0.95 & $0.81,1.12$ & 0.95 & $0.80,1.12$ & 0.91 & $0.76,1.08$ & 0.90 & $0.76,1.08$ \\
\hline Second highest quintile & 0.77 & $0.66,0.91^{\star *}$ & 0.77 & $0.65,0.90^{\star *}$ & 0.87 & $0.74,1.03$ & 0.86 & $0.73,1.02$ \\
\hline Highest quintile & 0.67 & $0.58,0.79^{\star \star \star}$ & 0.66 & $0.57,0.77^{\star \star \star}$ & 0.80 & $0.68,0.94^{\star \star}$ & 0.79 & $0.67,0.93^{\star \star}$ \\
\hline \multicolumn{9}{|l|}{ Religion } \\
\hline Muslim & & Ref. & & Ref. & & Ref. & & Ref. \\
\hline Other & $2 \cdot 28$ & $1.80,2.90^{\star \star \star}$ & 2.03 & $1.59,2.58^{\star \star \star}$ & 1.62 & $1 \cdot 25,2 \cdot 09^{\star \star \star}$ & 1.49 & $1.15,1.93^{\star *}$ \\
\hline \multicolumn{9}{|l|}{ Type of latrine } \\
\hline Improved $†$ & 0.98 & $0.85,1.12$ & 1.01 & $0.88,1 \cdot 15$ & 1.01 & $0.88,1.17$ & 1.05 & $0.90,1.21$ \\
\hline \multirow{2}{*}{\multicolumn{9}{|c|}{ Source of drinking-water }} \\
\hline & & & & & & & & \\
\hline Improved§ & 0.80 & $0.72,0.89^{\star \star \star}$ & 0.81 & $0.73,0.90^{\star \star \star}$ & 0.89 & $0.80,1.00$ & 0.91 & $0.81,1.01$ \\
\hline \multicolumn{9}{|l|}{ Remittance } \\
\hline No & & Ref. & & Ref. & & Ref. & & Ref. \\
\hline Yes & 1.07 & $0.94,1.21$ & 1.06 & $0.93,1.21$ & 1.11 & $0.98,1.27$ & $1 \cdot 11$ & $0.98,1.27$ \\
\hline \multicolumn{9}{|l|}{ Community } \\
\hline \multicolumn{9}{|c|}{ Time to upazila headquarters } \\
\hline$<30$ min & & & & Ref. & & & & Ref. \\
\hline $30-44 \min$ & & & 0.51 & $0.42,0.62^{* * *}$ & & & 0.60 & $0.49,0.73^{\star * *}$ \\
\hline$\geq 45 \mathrm{~min}$ & & & 0.67 & $0.55,0.82^{\star \star \star}$ & & & 0.78 & $0.64,0.97^{*}$ \\
\hline \multicolumn{9}{|c|}{ Availability of MOHFW or NGO clinic } \\
\hline No & & & & Ref. & & & & Ref. \\
\hline Yes & & & 1.03 & $0.83,1.28$ & & & 1.30 & $1.04,1.61^{\star}$ \\
\hline
\end{tabular}

RRR, relative risk ratio; NGO, non-governmental organization; MOHFW, Ministry of Health and Family Welfare; Ref., reference category. Model 1 examined the association of individual and household factors; model 2 additionally examined the effect of community variables. ${ }^{\star} P<0.05,{ }^{* \star} P<0.01,{ }^{* \star \star} P<0.001$.

tImproved latrine included all flushed and pit latrines with slab.

$\ddagger$ Non-improved latrine included pit latrine without slab, hanging latrine, dry latrine and no latrine/bush/field.

§lmproved sources of drinking-water included water from pipe/tap, tube well and tank.

||Non-improved sources of drinking-water included water from dug well, spring, rain and river/dam/lake/pond/stream/canal.

early stage of experiencing the dual burden of under- and overnutrition. Continuing underweight and increasing burden of overweight/obesity is a common phenomenon of rapidly growing economies ${ }^{(5)}$ where socio-economic disparities remain high $^{(7)}$. Underweight and overweight/ obesity are a result from an imbalance in the amounts of nutrients and energy required and consumed by the body. Underweight is associated with insufficient intakes of foods and nutrients and burden of infection that can perpetuate underweight status. On the other hand, among the higher socio-economic groups, food consumption is much higher and they also have a sedentary lifestyle, leading to overweight/obesity.
The present study provides evidence that while the underweight rate in Bangladesh has declined over the past 20 years, the rate remains high. The underweight rate reduced from $68.0 \%$ in $1993^{(29)}$ to $30 \cdot 1 \%$ in 2011 (using an underweight cut-off of $\mathrm{BMI} \leq 18.0 \mathrm{~kg} / \mathrm{m}^{2}$ ) among rural women of reproductive age. Another study conducted in 1994 among urban women living in slums of Bangladesh documented an underweight rate of $59.2 \%$ using the underweight cut-off of BMI $\leq 18.0 \mathrm{~kg} / \mathrm{m}^{2(30)}$.

Our findings that socio-economic variables are important determinants of nutritional status are similar to those of earlier studies examining these associations in Bangladesh. Household wealth status ${ }^{(22,27,28)}$ and higher 
educational attainment ${ }^{(7,27-29,31,36,39,40)}$ are wellestablished determinants of nutritional status. Like ours, earlier studies also reported that women in households with low socio-economic status experience a greater risk of underweight status and those in households with high socio-economic status experience a higher risk of being overweight/obese $^{(27,41)}$. The association suggests that women from poorer households may not afford sufficient foods to maintain their nutrition or experience higher rates of infections. On the other hand, no or low levels of education may be associated with lack of awareness about a relatively less expensive balanced diet that may result in undernutrition in women ${ }^{(26)}$. Our findings agree that both wealth and literacy are related to food security and dietary diversity ${ }^{(26)}$ of a household and thereby attribute to maternal underweight and overweight/obesity.

Food availability during the post-harvest period was found to be significantly associated with lower underweight rate. This is consistent with the finding of an earlier study on food insecurity in relation to nutritional status in Bangladesh $^{(26)}$. Non-Muslim women in Bangladesh are less likely to be overweight or obese, a finding also observed earlier ${ }^{(28)}$. A possible explanation for this could relate to social capital and limited resource access for religious minorities ${ }^{(28,29)}$. Concordant with results from other studies, household remittance was found to be significantly associated with lower risk of underweight, suggesting a relationship between remittance, social and economic capital, and improvements to family health status $^{(42-44)}$.

The increased likelihood of being underweight and decreased risk of being overweight/obese among younger women may be partly because of their awareness of being slim, their higher physical activity and their dietary habits. Berkel et al. discussed that individual behaviours, such as physical activity and good dietary practices, contribute to weight loss ${ }^{(45)}$. On the other hand, the likelihood of being underweight among the oldest women may be because of a cohort effect, as nutritional status has improved over time. The likelihood of being overweight/obese among the older group of women may partly be attributed to less physical activity ${ }^{(45)}$.

NGO membership was associated with higher likelihood of being underweight in unadjusted analysis, which disappeared when accounting for other covariates. This crude association could be due to a selection bias, because NGO often target women from very-low-income households presumably with lower nutritional status. A study of longitudinal nature might elucidate if active participation in NGO programmes can contribute to a decrease of underweight status of women over time. Another study found NGO presence to be related to better nutritional status, although more so in children than mothers ${ }^{(46)}$. Longer travel time to upazila headquarters was found to be significantly associated with underweight of women of childbearing age; there was a slight significant increase in likelihood of underweight for those who lived 31-44 min away rather than over $45 \mathrm{~min}$ away; however, the difference in the RRR is rather small and thus is not of practical significance.

Our findings of lower risk of stunting in women with secondary education and higher household wealth are consistent with the literature including from Bangla$\operatorname{desh}^{(9-13,47)}$. Adult height is determined by genetic predispositions and environmental factors ${ }^{(11)}$. In addition to genetic influence, income, social status, infection and nutrition were shown to affect body height in the European general population ${ }^{(47)}$. Environmental factors are likely to be more important determinants of height in lowand middle-income countries since environmental stress including food availability and infections is much higher in such countries compared with high-income countries ${ }^{(10,11)}$. Perkins et al. explained in their review that short adult stature in low- and middle-income countries is mainly because of the cumulative net impact of nutrition associated with disease and environmental conditions, such as socio-economic status ${ }^{(10)}$.

Use of improved drinking-water was associated with lower risk of stunting. Improved water may be a proxy for less exposure to enteric pathogens. Watanabe and Petri discussed that environmental enteropathy is a chronic disease caused by continuous exposure to faecally contaminated food and water that does not produce symptoms but contributes to poor physical development ${ }^{(48)}$.

The present study has several limitations. Inferences should be limited due to the cross-sectional nature of the study. As data on height, weight and other covariates were collected simultaneously, understanding a causal relationship of the factors on nutritional status is not possible due to a lack of temporality. Additionally, reverse causational associations are possible between factors such as nutrition status and educational attainment, NGO membership and wealth levels. We were not able to examine several risk factors such as household food security, micronutrient intakes, physical activity, media exposure and decision-making ability, which are important components for nutritional assessment of women of childbearing age. However, we created a proxy variable for food availability at the community level and demonstrated a lower rate of underweight status in the post-harvest season. Also, the study did not include information on, for example, anaemia, infection (malaria, dengue and HIV) and management of illness, which might be important for nutritional assessment of women.

The strength of the study is that it was large, populationbased and restricted to non-pregnant women. We examined different levels of variables that may affect malnutrition among women. Future studies could address issues of temporality with a longitudinal design and incorporate additional relevant variables that were not included herein. 
Bangladesh has experienced a substantial reduction of underweight status in women of childbearing age; however, the underweight rate still remains high, with an emergence of overweight/obesity among women. Maternal underweight contributes to fetal growth restriction, which increases the risk of stillbirth and neonatal death. Overweight/obesity in women is associated with increased risk of chronic diseases, such as hypertension, diabetes and CVD, as well as with complications during pregnancy, labour and postpartum, such as gestational diabetes mellitus, pre-eclampsia, maternal death and haemorrhage ${ }^{(49)}$. To combat the underweight, overweight/obesity and stunting of women of childbearing age, Bangladesh requires multidimensional intervention programmes based on identified individual-, householdand community-level sociodemographic and economic risk factors that affect maternal nutritional status. A Bangladesh health, population and nutrition sector programme already has the following interventions to promote women's nutrition: counselling on adequate nutrition during antenatal and postnatal contacts; and provision of iron-folic acid supplements to pregnant women. Bangladesh may consider replacing iron-folic acid by multiple-micronutrient supplements to all pregnant women, provision of calcium supplementation to those at risk of low intake and provision of balanced energy-protein supplementation to pregnant women as needed, as recommended in the second Lancet series on maternal and child nutrition ${ }^{(50)}$. Regular systematic monitoring and surveillance of the social trajectory of nutritional status is essential to develop an appropriate strategy to reduce the dual burden of malnutrition in Bangladesh.

\section{Acknowledgements}

Acknowledgements: The authors acknowledge the contribution of the study women and the dedication of the Projahnmo field team. Projahnmo is a research partnership of Johns Hopkins University, the Bangladesh MOHFW and other Bangladeshi institutions including icddr,b and Shimantik. Financial support: This study was supported by the Eunice Kennedy Shriver National Institute of Child Health and Human Development (NICHD; grant number R01 HD066156-02). The NICHD had no role in the design, data collection, analysis or interpretation; or manuscript preparation and submission. Conflict of interest: The authors declare that they have no competing interests. Authorship: R.K. and A.H.B. conceived and designed the analysis. A.H.B., A.S.C.C.L., M.A.Q. and L.C.M. designed and implemented the parent project. L.C.M. and N.B. developed and maintained the database. R.K. and M.R. conducted data analyses. R.K. drafted the first version of the manuscript. All authors read, provided technical input and approved the final manuscript. Ethics of buman subject participation: Ethical approval for the collection of data was obtained from the Johns Hopkins University Institutional Review Board and the icddr,b Ethical Review Committee.

\section{References}

1. Bhutta ZA, Ahmed T, Black RE et al. (2008) What works? Interventions for maternal and child undernutrition and survival. Lancet 371, 417-440.

2. Black RE, Allen LH, Bhutta ZA et al. (2008) Maternal and child undernutrition: global and regional exposures and health consequences. Lancet 371, 243-260.

3. Victora CG, Adair L, Fall C et al. (2008) Maternal and child undernutrition: consequences for adult health and human capital. Lancet 371, 340-357.

4. Bryce J, Coitinho D, Darnton-Hill I et al. (2008) Maternal and child undernutrition: effective action at national level. Lancet 371, 510-526.

5. Black RE, Victora CG, Walker SP et al. (2013) Maternal and child undernutrition and overweight in low-income and middle-income countries. Lancet 382, 427-451.

6. Ahmed T, Mahfuz M, Ireen S et al. (2012) Nutrition of children and women in Bangladesh: trends and directions for the future. J Health Popul Nutr 30, 1-11.

7. Kamal SMM, Hassan CH \& Alam GM (2015) Dual burden of underweight and overweight among women in Bangladesh: patterns, prevalence, and sociodemographic correlates. $J$ Health Popul Nutr 33, 92-105.

8. Osmani S \& Sen A (2003) The hidden penalties of gender inequality: fetal origins of ill-health. Econ Hum Biol 1, 105121.

9. Bogin B, Scheffler C \& Hermanussen M (2017) Global effects of income and income inequality on adult height and sexual dimorphism in height. Am J Hum Biol 29, e22980.

10. Perkins JM, Subramanian SV, Davey Smith G et al. (2016) Adult height, nutrition, and population health. Nutr Rev $\mathbf{7 4}$, 149-165.

11. Silventoinen K (2003) Determinants of variation in adult body height. J Biosoc Sci 35, 263-285.

12. de Oliveira VH \& Quintana-Domeque C (2014) Early-life environment and adult stature in Brazil: an analysis for cohorts born between 1950 and 1980. Econ Hum Biol 15, 67-80.

13. National Institute of Population Research and Training, Mitra and Associates, \& ICF International (2014) Bangladesh Demographic and Health Survey 2014. Dhaka and Rockville, MD: NIPORT, Mitra and Associates, and ICF International.

14. Shafique S, Akhter N, Stallkamp G et al. (2007) Trends of under- and overweight among rural and urban poor women indicate the double burden of malnutrition in Bangladesh. Int J Epidemiol 36, 449-457.

15. Mallia T, Grech A, Hili A et al. (2017) Genetic determinants of low birth weight. Minerva Ginecol 69, 631-643.

16. Dahlui M, Azahar N, Oche OM et al. (2016) Risk factors for low birth weight in Nigeria: evidence from the 2013 Nigeria Demographic and Health Survey. Glob Health Action 9, 28822.

17. Nnam NM (2017) Improving maternal nutrition for better pregnancy outcomes. Proc Nutr Soc 74, 454-459.

18. Khan MN, Rahman MM, Shariff AA et al. (2017) Maternal undernutrition and excessive body weight and risk of birth and health outcomes. Arch Public Health 75, 12.

19. Xu H, Shatenstein B, Luo Z-C et al. (2009) Role of nutrition in the risk of preeclampsia. Nutr Clin Care 67, 639-657.

20. Chopra M, Galbraith S \& Darnton-Hill I (2002) A global response to a global problem: the epidemic of overnutrition. Bull World Health Organ 80, 952-958. 
21. Ng M, Fleming T, Robinson M et al. (2014) Global, regional and national prevalence of overweight and obesity in children and adults 1980-2013: a systematic analysis. Lancet 384, 766-781.

22. Zahangir MS, Hasan MM, Richardson A et al. (2017) Malnutrition and non-communicable diseases among Bangladeshi women: an urban-rural comparison. Nutr Diabetes $\mathbf{7}$, e250.

23. Ly KA, Ton TGN, Ngo QV et al. (2013) Double burden: a cross-sectional survey assessing factors associated with underweight and overweight status in Danang, Vietnam. BMC Public Health 13, 35.

24. Fenn B, Bulti AT, Nduna T et al. (2012) An evaluation of an operations research project to reduce childhood stunting in a food-insecure area in Ethiopia. Public Health Nutr 15, $1746-1754$

25. World Health Organization, UNICEF \& US Agency for International Development (2015) Improving Nutrition Outcomes with Better Water, Sanitation and Hygiene: Practical Solutions for Policy and Programmes. Geneva: WHO.

26. Harris-Fry H, Azad K, Kuddus A et al. (2015) Socioeconomic determinants of household food security and women's dietary diversity in rural Bangladesh: a crosssectional study. J Health Popul Nutr 33, 12.

27. Islam A, Islam N, Bharati P et al. (2016) Socio-economic and demographic factors influencing nutritional status among early childbearing young mothers in Bangladesh. BMC Womens Health 16, 58.

28. Hossain MG, Bharati P, Aik SAW et al. (2012) Body mass index of married Bangladeshi women: trends and association with socio-demographic factors. J Biosoc Sci 44, 385399.

29. Bhuiya A \& Mostafa G (1993) Levels and differentials in weight, height and body mass index among mothers in a rural area of Bangladesh. J Biosoc Sci 25, 31-38.

30. Baqui AH, Arifeen SE, Amin S et al. (1994) Levels and correlates of maternal nutrition status in urban Bangladesh. Eur J Clin Nutr 48, 349-357.

31. Ahsan KZ, Arifeen SE, Al-Mamun MA et al. (2017) Effects of individual, household and community characteristics on child nutritional status in the slums of urban Bangladesh. Arch Public Health 75, 9.

32. Baqui AH, El-Arifeen S, Darmstadt GL et al. (2008) Effect of community-based newborn-care intervention package implemented through two service-delivery strategies in Sylhet district, Bangladesh: a cluster-randomised controlled trial. Lancet 371, 1936-1944.

33. Arifeen SE, Mullany LC, Shah R et al. (2012) The effect of cord cleansing with chlorhexidine on neonatal mortality in rural Bangladesh: a community-based, clusterrandomised trial. Lancet 379, 1022-1028.

34. Lee ACC, Quaiyum MA, Mullany LC et al. (2015) Screening and treatment of maternal genitourinary tract infections in early pregnancy to prevent preterm birth in rural Sylhet, Bangladesh: a cluster randomized trial. BMC Pregnancy Childbirth 15, 326.

35. Andridge RR (2011) A review of hot deck imputation for survey non-response. Int Stat Rev 78, 40-64.

36. Mohsena M, Goto R \& Mascie-Taylor CN (2016) Maternal nutritional status (as measured by height, weight and BMI) in Bangladesh: trends and socio-economic association over the period 1996 to 2007. Public Health Nutr 19, 14381445.

37. Sengupta A, Angeli F, Syamala TS et al. (2014) State-wise dynamics of the double burden of malnutrition among 1549 year-old women in India: how much does the scenario change considering Asian population-specific BMI cut-off values? Ecol Food Nutr 53, 618-638.

38. Bharati S, Pal M, Bhattacharya BN et al. (2007) Prevalence and causes of chronic energy deficiency and obesity in Indian women. J Hum Biol 79, 395-412.

39. Rengma MS, Sen J \& Mondal N (2015) Socio-economic, demographic and lifestyle determinants of overweight and obesity among adults of northeast India. Ethiop J Health Sci 25, 199-208.

40. Ahmed SM, Adams A, Chowdhury AM et al. (1998) Chronic energy deficiency in women from rural Bangladesh: some socioeconomic determinants. J Biosoc Sci 30, 349-358.

41. Subramanian SV \& Smith GD (2006) Patterns, distribution, and determinants of under- and overnutrition: a populationbased study of women in India. Am J Clin Nutr 84, 633-640.

42. Lu Y (2012) Household migration, remittances, and its impact on health in Indonesia. Int Migr 51, Suppl. 1, doi: 10.1111/j.1468-2435.2012.00761.x

43. Hildebrandt N \& McKenzie D (2005) The effects of migration on child health in Mexico (with comments). Economia 6, 257-289.

44. Anton J-I (2010) The impact of remittances on nutritional status of children in. Int Migr Rev 44, 269-299.

45. Berkel LA, Poston WSC, Reeves RS et al. (2005) Behavioral interventions for obesity. J Am Diet Assoc 105, 35-43.

46. Linnemayr S, Alderman H \& Ka A (2008) Determinants of malnutrition in Senegal: individual, household, community variables, and their interaction. Econ Hum Biol 6, 252-263.

47. Fudvoye J \& Parent AS (2017) Secular trends in growth. Ann Endocrinol (Paris) 78, 88-91.

48. Watanabe K \& Petri WA Jr (2016) Environmental enteropathy: elusive but significant subclinical abnormalities in developing countries. EBioMedicine 10, 25-32.

49. Van Lieshout RJ, Taylor VH \& Boyle MH (2011) Prepregnancy and pregnancy obesity and neurodevelopmental outcomes in offspring: a systematic review. Obes Rev 12, 548-559.

50. Bhutta ZA, Das JK, Rizvi A et al. (2013) Evidence-based interventions for improvement of maternal and child nutrition: what can be done and at what cost? Lancet 382, 452477. 